\title{
Synthesis and properties of epitaxial thin films of $c$-axis oriented metastable four-layered hexagonal $\mathrm{BaRuO}_{3}$
}

\author{
M. K. Lee and C. B. Eom ${ }^{\text {a) }}$ \\ Department of Mechanical Engineering and Materials Science, Duke University, Durham, \\ North Carolina 27708 \\ W. Tian and X. Q. Pan \\ Department of Materials Science and Engineering, University of Michigan, Ann Arbor, Michigan 48109 \\ M. C. Smoak and F. Tsui \\ Department of Physics and Astronomy, University of North Carolina, Chapel Hill, North Carolina 27599 \\ J. J. Krajewski \\ Bell Laboratories, Lucent Technologies, Murray Hill, New Jersey 07974
}

(Received 4 January 2000; accepted for publication 15 May 2000)

\begin{abstract}
We have grown epitaxial thin films of $c$-axis oriented metastable four-layered hexagonal $\mathrm{BaRuO}_{3}$ on a (111) $\mathrm{SrTiO}_{3}$ substrate by $90^{\circ}$ off-axis sputtering techniques. X-ray diffraction and transmission electron microscopy reveal that the films are single domains of $c$-axis four-layered hexagonal structures with an in-plane epitaxial arrangement of $\mathrm{BaRuO}_{3}[2 \overline{1} \overline{1} 0] \| \mathrm{SrTiO}_{3}[110]$. Surfaces with smooth terraces having a step height of a half unit cell $(\sim 4.7 \AA)$ have been observed by scanning tunneling microscopy. The in-plane electrical resistivity of the films is metallic, with a room temperature value of $810 \mu \Omega \mathrm{cm}$ and slightly curved temperature dependence. Their magnetic susceptibility is Pauli paramagnetic. The metastable layered metallic oxide can be used for understanding new solid-state phenomena and device applications. () 2000 American Institute of Physics. [S0003-6951(00)00228-X]
\end{abstract}

The recent discovery of superconductivity in the $\mathrm{Sr}_{2} \mathrm{RuO}_{4}$ compound without any copper and doping ${ }^{1}$ has led to renewed interest in the physical properties, particularly magnetism, and structural chemistry of the ruthenium based oxides $\left(A \mathrm{RuO}_{3}: A=\mathrm{Ba}, \mathrm{Sr}, \mathrm{Ca}\right)$. Among all ruthenates, only $\mathrm{BaRuO}_{3}$, which has the largest $A$-site cation in the series, possesses a hexagonal structure in the bulk, while others have a $\mathrm{GdFeO}_{3}$-type orthorhombic structure instead. ${ }^{2}$

The structural chemistry of $\mathrm{ABO}_{3}$-type ruthenates can be described in terms of hexagonal- and cubic-close packing of $\mathrm{AO}_{3}$ layers. If all $\mathrm{AO}_{3}$ layers are cubic-close packed, the $\mathrm{RuO}_{6}$ octahedra form a cubic-like three-dimensional array by sharing only one oxygen to give rise to cubic, tetragonal, and orthorhombic structures. In contrast, if $\mathrm{AO}_{3}$ layers are entirely hexagonal-close packed, the $\mathrm{RuO}_{6}$ octahedra are shared by three oxygens to form a hexagonal structure. Due to the two basic packing forms, it is recognized that the bulk $\mathrm{BaRuO}_{3}$ ceramic has three different crystal structures. They are (1) the nine-layered rhombohedral structure $(9 R)$ with $a=5.75 \AA$ and $c=21.6 \AA{ }^{3}$ (2) the six-layered hexagonal structure $(6 H)$ with $a=5.71 \AA$ and $c=14 \AA,{ }^{4}$ and (3) the four-layered hexagonal structure $(4 H)$ with $a=5.73 \AA$ and $c=9.5 \AA,{ }^{4}$ depending on the amount of hexagonal- and cubic-close packing of the $\mathrm{BaO}_{3}$ layers. The $9 R$ phase has been considered the most stable form in the bulk. ${ }^{3}$ Recently, the synthesis of epitaxial thin films of $9 R \mathrm{BaRuO}_{3}$ with $(20 \overline{2} 5)$ texture has been reported. ${ }^{5}$ The $6 H$ phase has been found mainly as a result of high-pressure bulk synthesis. Likewise, the $4 \mathrm{H}$ structure containing pure $\mathrm{BaRuO}_{3}$ has not

${ }^{a)}$ Electronic mail: eom@acpub.duke.edu been synthesized reproducibly under ordinary conditions either, so it has been considered as metastable. The first bulk ceramic $4 \mathrm{HBaRuO}_{3}$ was synthesized under high pressure (between 15 and 30 kbar) by Longo and Kafalas, ${ }^{4}$ and its single crystal form was grown using a $\mathrm{BaCl}_{2}$ flux. ${ }^{6}$ More recently, the electrical and magnetic properties of bulk single crystal form have been measured, ${ }^{7}$ although its synthesis is not reproducible.

In this letter, we report the synthesis, electrical transport, and magnetic properties of epitaxial thin films of metastable $4 \mathrm{H} \mathrm{BaRuO}_{3}$. In order to match the in-plane symmetry and lattice of (0001) $4 \mathrm{H} \mathrm{BaRuO}_{3}$, the following substrates have been chosen: (0001) $\mathrm{Al}_{2} \mathrm{O}_{3}$, (111) $\mathrm{SrTiO}_{3}$, (111) $\mathrm{MgAl}_{2} \mathrm{O}_{4}$, and (111) $\mathrm{MgO}$. The films were grown by $90^{\circ}$ off-axis sputtering techniques ${ }^{8}$ from a stoichiometric $\mathrm{BaRuO}_{3}$ ceramic target. The total pressure of the oxygen and argon mixture was maintained at 200 mTorr. $\mathrm{O}_{2}$ partial pressures of 8 and 80 mTorr were used, but except for the resistivity no apparent effects on either the crystal structure or the growth mode were observed. The substrate block temperature was held at $650{ }^{\circ} \mathrm{C}$. After deposition, the samples were cooled down to room temperature in an oxygen pressure of 300 Torr. Results reported here are for films $4600 \AA$ thick.

Their crystal structures and epitaxial arrangements were studied by $\mathrm{x}$-ray diffraction and transmission electron microscopy (TEM). All films studied exhibit predominantly $c$-axis texture normal to the substrates. The films grown on (111) $\mathrm{MgAl}_{2} \mathrm{O}_{4}$ substrates exhibit two hexagonal domains, rotated by about $4^{\circ}$ with respect to each other in the growth plane, and they also contain a small amount of impurity phases. It is considered that the spinel structure of the 

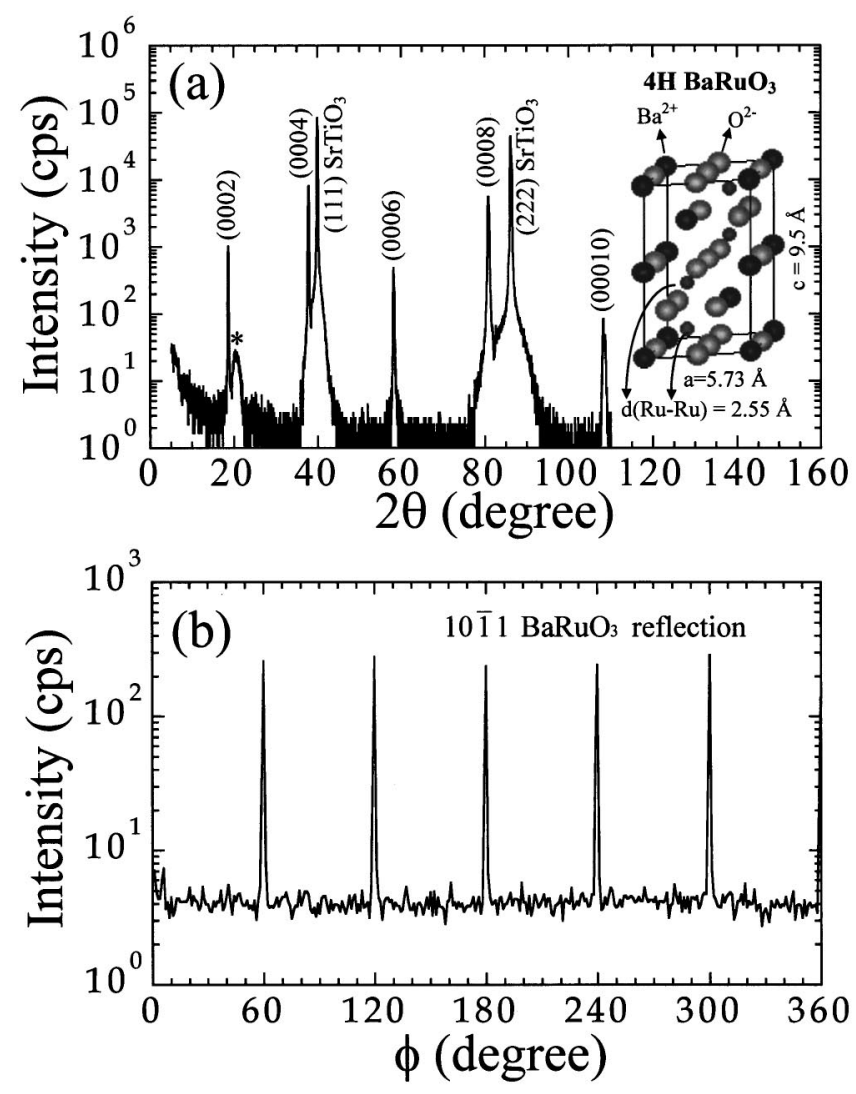

FIG. 1. X-ray diffraction patterns of a $4600 \AA \mathrm{BaRuO}_{3}$ thin film grown on a (111) $\mathrm{SrTiO}_{3}$ substrate: (a) normal $\theta-2 \theta$ scan (the inset shows a schematic diagram of the $4 \mathrm{H} \mathrm{BaRuO}_{3}$ structure) and (b) azimuthal off-axis $\phi$ scan for the (1011) $\mathrm{BaRuO}_{3}$ reflection. The bremsstrahlung peak from the substrate is labeled by "**,"

$\mathrm{MgAl}_{2} \mathrm{O}_{4}$ substrate does not match structurally with the $4 \mathrm{HBRuO}_{3}$ phase, even though the lattice spacing along [220] of the (111) $\mathrm{MgAl}_{2} \mathrm{O}_{4}$ plane matches well with the basal plane of $4 \mathrm{H} \mathrm{BaRuO}_{3}$ (mismatch: $+0.24 \%$ ). The films grown on (0001) $\mathrm{Al}_{2} \mathrm{O}_{3}$ substrates (mismatch: + 20.41\%) also contain a considerable amount of (110) $4 H$ texture. These films and their counterparts grown on (111) $\mathrm{MgO}$ with a mismatch of $-3.91 \%$ and a Rochsalt structure also contain impurity phases, which have not yet been determined. In contrast, the films grown on (111) $\mathrm{SrTiO}_{3}$ (mismatch: $+3.73 \%$ ) consist of purely single domains of (0001) $4 H$ phase, as shown in Fig. 1(a). This is consistent with the fact that the (111) $\mathrm{SrTiO}_{3}$ matches with (0001) $4 \mathrm{H} \mathrm{BaRuO}_{3}$, both in terms of chemical compatibility, particularly for being a perovskite also, and lattice parameters.

Lattice parameters of the films are determined by normal $\theta-2 \theta$ scan and grazing incidence diffraction (GID) techniques. For a $4600 \AA$ thick film grown on (111) $\mathrm{SrTiO}_{3}$, the values are $9.50 \pm 0.01 \AA$ out of plane and $5.728 \pm 0.002 \AA$ in plane, both of which are the same as for the bulk. The inplane epitaxial arrangement is determined by the azimuthal $\phi$ scans of both $\mathrm{BaRuO}_{3} 10 \overline{1} 1$ [Fig. 1(b)] and $\mathrm{SrTiO}_{3} 002$ reflections (not shown here). The observed sixfold intensities of the $\mathrm{BaRuO}_{3} 10 \overline{1} 1$ reflection confirm that the film contains only a single hexagonal texture in the plane. The azimuthal scans also indicate that $\mathrm{BaRuO}_{3}[2 \overline{1} \overline{1} 0]$ is parallel to $\mathrm{SrTiO}_{3}$ [110]. The measured full width at half maximum (FWHM)
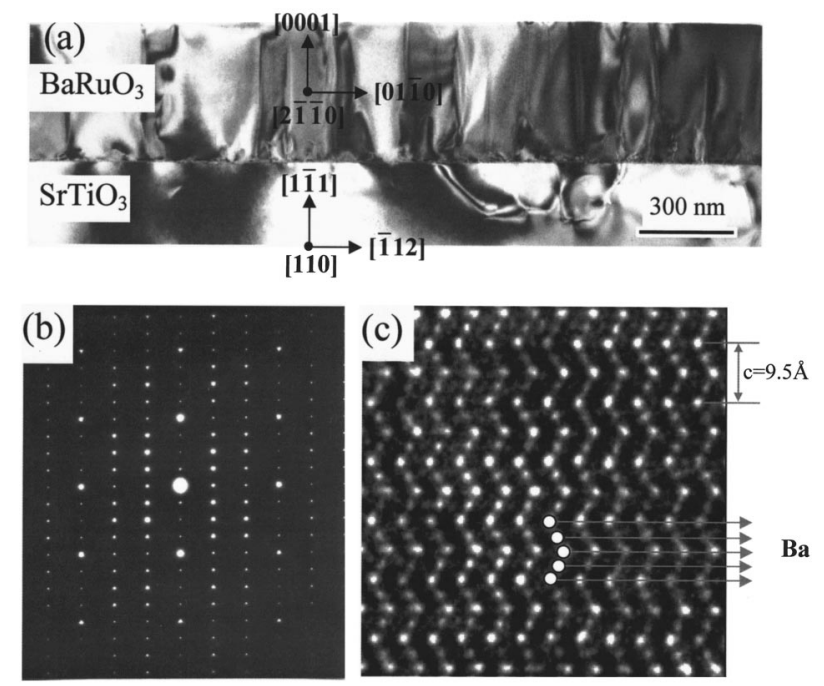

FIG. 2. Cross-sectional TEM of a $4600 \AA \mathrm{BaRuO}_{3}$ film grown on a (111) $\mathrm{SrTiO}_{3}$ substrate: (a) cross-sectional bright field image, (b) selected area electron diffraction pattern, and (c) high resolution image taken from the $[2 \overline{1} \overline{1} 0]$ zone axis of the film. The four-layered stacking of $\mathrm{BaRuO}_{3}$ is shown clearly in (b) and (c).

of the rocking curve of the 0004 reflection is $0.39^{\circ}$ and that of the azimuthal $\phi$ scan of the $10 \overline{1} 1$ reflection is $0.37^{\circ}$.

The microstructure has been studied further by crosssectional TEM, including selected area electron diffraction (SAED). Figure 2(a) is a low magnification bright field TEM image of a $4600 \AA$ thick $\mathrm{BaRuO}_{3}$ film grown on a (111) $\mathrm{SrTiO}_{3}$ substrate. The dark bands indicate the presence of antiphase boundaries and stress-induced dislocations. The latter is typical for an epitaxial system with large lattice mismatch. Figure 2(b) and 2(c) show a SAED pattern and a high resolution image, respectively, taken from the [2 $\overline{1} \overline{1} 0]$ zone axis of the film. These results further confirm that the $\mathrm{BaRuO}_{3}$ film consists of a single domain $4 \mathrm{H}$ structure, grown epitaxially along the $c$ axis on the (111) $\mathrm{SrTiO}_{3}$ substrate. The lattice constants determined by TEM are 5.72 $\pm 0.08 \AA$ in plane and $9.53 \pm 0.07 \AA$ out of plane, consistent with those obtained by x-ray diffraction.

The growth mechanism and surface morphologies have been studied by scanning tunneling microscopy (STM). The films grown on the (111) $\mathrm{SrTiO}_{3}$ substrate exhibit large concentric "birthday cake-like" terraces about 1000-2500 ̊ in diameter, leading to a relatively small roughness, as illustrated in Fig. 3(a). The root-mean-squared (rms) roughness is about $8-10 \AA$ over $5 \times 5 \mu \mathrm{m}$ area. The observed uniformly distributed three-dimensional surface morphology indicates a two-dimensional island growth. The measured step height between the concentric terraces is about $4.7 \AA$, as shown in Fig. 3(b), which is one half of the $c$-axis lattice parameter of $4 \mathrm{HaRuO}_{3}$.

Figure 4 shows the in-plane electrical resistivity as a function of temperature for a $4600 \AA$ film grown at a high $\mathrm{O}_{2}$ pressure ( $80 \mathrm{mTorr})$. The room temperature and residual resistivities are 810 and $220 \mu \Omega \mathrm{cm}$, respectively, and they are nearly twice the values of a bulk single crystal, i.e., 400 and $100 \mu \Omega \mathrm{cm} .{ }^{7}$ The higher values observed in the film can be attributed to the presence of antiphase boundaries and misfit dislocations described above [see Fig. 2(a)]. The temperature dependence of the measured resistivity is metallic down to 
(a)

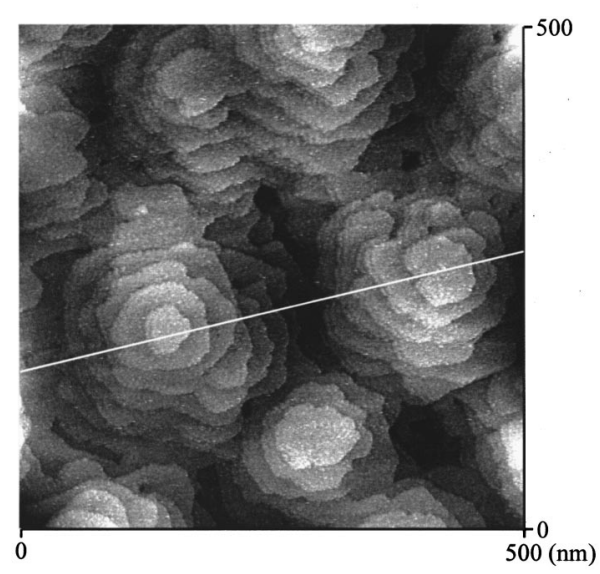

(b)

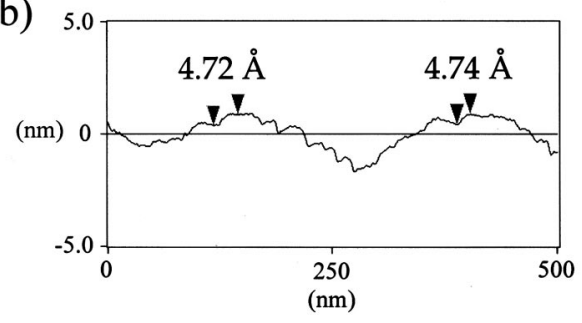

FIG. 3. (a) STM image of a (0001) $4 \mathrm{H} \mathrm{BaRuO}_{3}$ thin film grown on (111) $\mathrm{SrTiO}_{3}$ and (b) section analysis of the image along the line drawn in (a) to indicate the cross-sectional profile including the step height.

$4.2 \mathrm{~K}$ and it is slightly curved at low temperatures with a nearly quadratic dependence below $70 \mathrm{~K}$. The nearly quadratic behavior is illustrated in the Fig. 2 inset by the nearly linear $T^{2}$ dependence, with a slope of $2.8 \times 10^{-2} \mu \Omega \mathrm{cm} /$

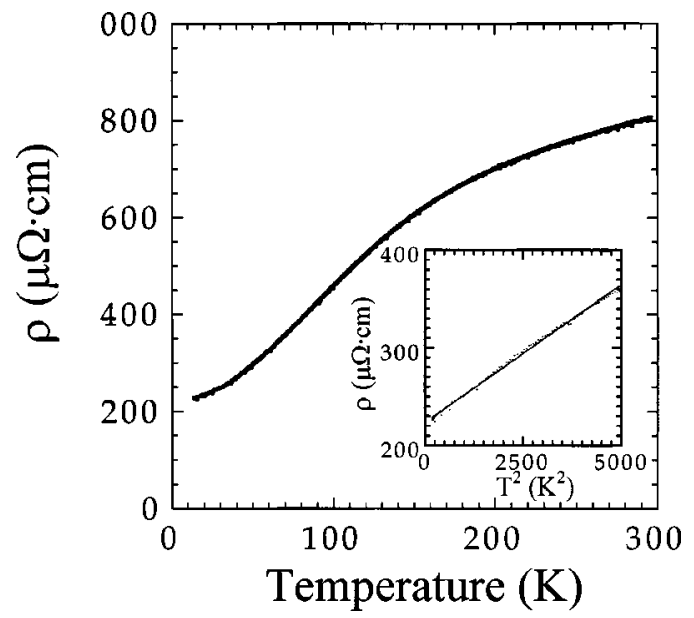

FIG. 4. In-plane electrical resistivity as a function of temperature of a 4600 $\AA$ (0001) $4 \mathrm{H} \mathrm{BaRuO}_{3}$ thin film grown on (111) $\mathrm{SrTiO}_{3}$. The inset illustrates the quadratic temperature dependence of the resistivity at low temperatures.
$\mathrm{K}^{2}$. The behavior is similar to that of the bulk $4 H$ single crystal, ${ }^{7}$ but it is different from that of the $9 R$ single crystal, which exhibits a transition from metallic at high temperature to a more resistive state at low temperature. ${ }^{7}$ The films grown at a lower $\mathrm{O}_{2}$ pressure $(8 \mathrm{mTorr})$ exhibit higher resistivites $\left(\rho_{295 \mathrm{~K}}=2100-2400 \mu \Omega \mathrm{cm}\right)$, which might be due to oxygen deficiency.

Magnetic properties of the $4 \mathrm{H} \mathrm{BaRuO}_{3}$ films have been studied by superconducting quantum interference device (SQUID) magnetometry. The susceptibility is paramagnetic and nearly temperature independent, indicating that it is dominated by Pauli paramagnetism. The measured susceptibility is $(8 \pm 2) \times 10^{-5} \mathrm{emu} / \mathrm{cm}^{3}$. These are also comparable to those observed in the bulk. ${ }^{7}$

In summary, we have synthesized metastable epitaxial $4 \mathrm{HaRuO}_{3}$ thin films, and have subsequently studied their structural, electrical transport, and magnetic properties. The $\mathrm{BaRuO}_{3}$ films grown on (111) $\mathrm{SrTiO}_{3}$ exhibit a purely $c$-axis single domain $4 H$ structure with good crystalline qualities and smooth surfaces. These metastable epitaxial thin films can help to understand new phenomena such as superconductivity in layered compounds without any copper oxide layers. ${ }^{1,7}$ They also show promise as metallic templates for the synthesis and characterization of epitaxial heterostructures for device applications, particularly for growth of (111) perovskites and $c$-axis hexagonal oxides. For instance, a (0001) $\mathrm{BaRuO}_{3}$ electrode layer with a smooth surface may allow the growth of (111) oriented ferroelectric heterostructures that require a high crystalline quality and sharp interfaces.

This work was supported by ONR Grant No. N0001495-1-0513, NSF Grant No. DMR-980244, DMR-9973801, a NSF Young Investigator Award, to one of the authors (C.B.E.), a David and Lucille Packard fellowship to the same author, a College of Engineering at the University of Michigan grant to another author (X.Q.P.), and NSF, Grant Nos. DMR-9703419 and DMR-9601825 to a third author (F.T.).

${ }^{1}$ Y. Maeno, H. Hashimoto, K. Yoshida, S. Nishizaki, T. Fujita, J. G. Bednorz, and F. Lichtenberg, Nature (London) 372, 532 (1994).

${ }^{2}$ H. Kobayashi, M. Nagata, R. Kanno, and Y. Kawamoto, MRS Bull. 29, 1271 (1994).

${ }^{3}$ P. C. Donohue, L. Katz, and R. Ward, Inorg. Chem. 4, 306 (1965).

${ }^{4}$ J. M. Longo and J. A. Kafalas, MRS Bull. 3, 687 (1968).

${ }^{5}$ J. Lettieri, I. W. Scrymgeour, D. G. Schlom, M. K. Lee, and C. B. Eom (unpublished).

${ }^{6}$ S. T. Hong and A. W. Sleight, J. Solid State Chem. 128, 251 (1997).

${ }^{7}$ J. T. Rijssenbeek, R. Jin, Y. Zadorozhny, Y. Liu, B. Batlogg, and R. J. Cava, Phys. Rev. B 59, 4561 (1999).

${ }^{8}$ C. B. Eom, R. J. Cava, R. M. Fleming, J. M. Phillips, R. B. van Dover, J. H. Marshall, J. W. P. Hsu, J. J. Krajewski, and W. F. Peck, Jr., Science 258, 1766 (1992). 